\title{
Competing Pathways for Nucleation of the Double Perovskite Structure in the Epitaxial Synthesis of $\mathrm{La}_{2} \mathrm{MnNiO}_{6}$
}

Steven R. Spurgeon, ${ }^{1}$ Yingge Du, ${ }^{1}$ Timothy Droubay, ${ }^{1}$ Arun Devaraj, ${ }^{1}$ Xiahan Sang, ${ }^{2}$ Paolo Longo, ${ }^{3}$ Pengfei Yan, ${ }^{4}$ Paul G. Kotula, ${ }^{5}$ Vaithiyalingam Shutthanandan, ${ }^{4}$ Mark

E. Bowden, ${ }^{4}$ James M. LeBeau, ${ }^{2}$ Chongmin Wang, ${ }^{4}$ Peter V. Sushko, ${ }^{1}$ and Scott A. Chambers $^{1}$

1) Physical and Computational Sciences Directorate, Pacific Northwest National Laboratory, Richland, WA, USA

${ }^{2)}$ Department of Materials Science and Engineering, North Carolina State University, Raleigh, NC, USA

3) Gatan Inc., Pleasanton, CA, USA

4) Environmental and Molecular Sciences Laboratory, Pacific Northwest National Laboratory, Richland, WA, USA

5) Sandia National Laboratories, Albuquerque, NM, USA 


\section{SUPPORTING INFORMATION}

\section{A. Film Structure and Composition}

In Figures S1.A-B we show reflection high-energy electron diffraction (RHEED) patterns measured along [100] crystallographic directions for a clean STO (001) substrate and an LMNO film of $\sim 40 \mathrm{~nm}$ thickness. The strong and streaky pattern of the film matches that of the STO, indicating epitaxy and a well-ordered surface. A high-resolution XRD pattern taken near the (002) Bragg reflection (see Figure S1.C) further confirms the epitaxial relationship. Fitting of the clearly resolved thickness fringes suggests a thickness of $\sim 40.5$ $\mathrm{nm}$, in good agreement with the nominal $40 \mathrm{~nm}$ value calculated from RHEED oscillations.
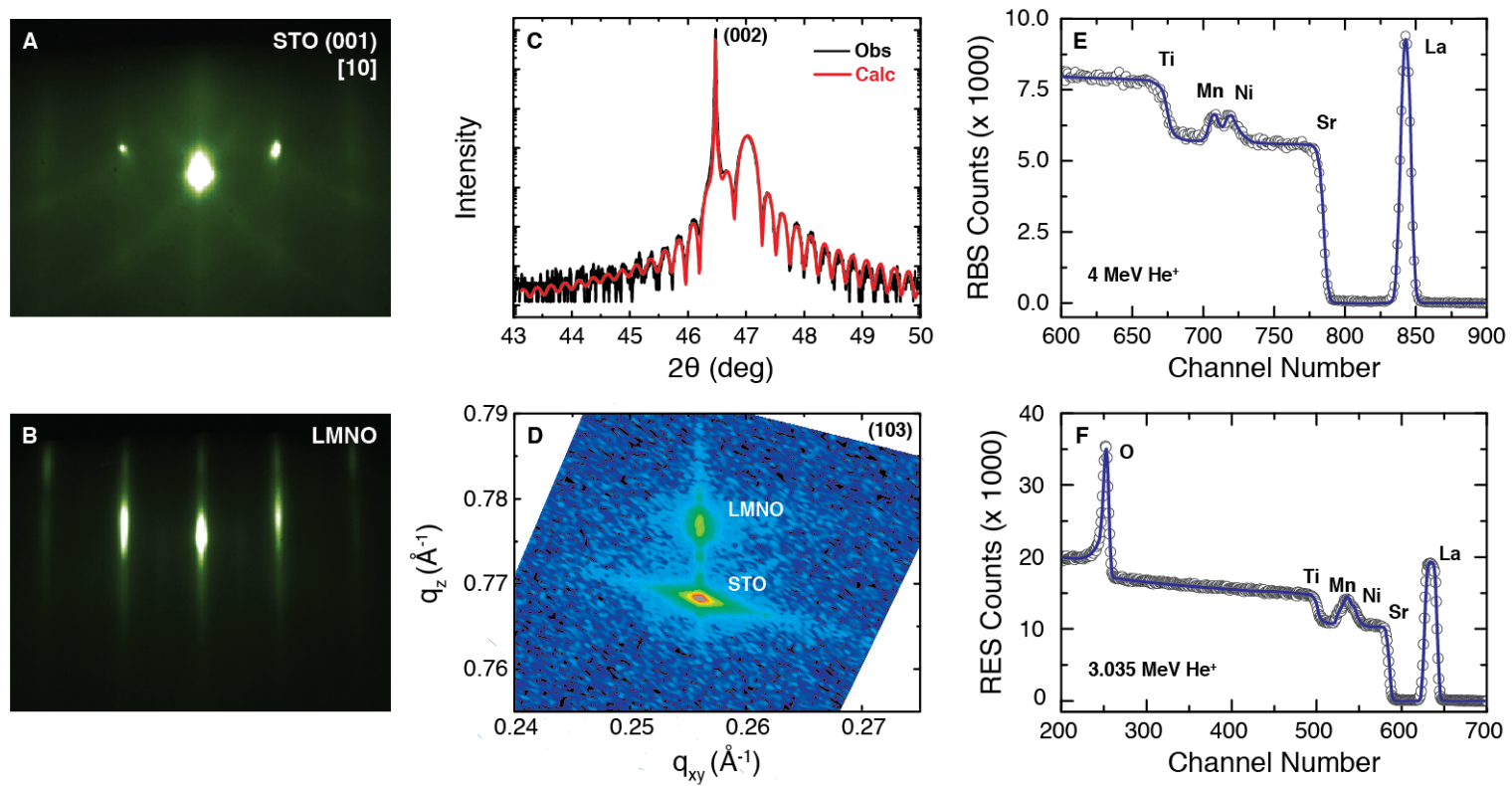

FIG. S1. Volume-averaged film structure and composition. (A, B) RHEED patterns taken along the [100] azimuthal direction for an STO (001) substrate and a LMNO film with a nominal thickness of $40 \mathrm{~nm}$, respectively. (C) High-resolution XRD pattern measured near the (002) Bragg peak for the $40 \mathrm{~nm}$ LMNO film. Fitting of the thickness fringes suggests a thickness of $\sim 40.5$ nm. (D) Reciprocal space map taken around the (103) film reflection, showing coherent in-plane strain. (E) RBS measurement at $4 \mathrm{MeV} \mathrm{He}^{+}$ion energy, overlaid with the calculated fit to different species. (F) RES measurement at $3.035 \mathrm{MeV}$ at $\mathrm{He}^{+}$ion energy, showing a strongly enhanced $\mathrm{O}$ resonance peak. 
Coherent in-plane strain is revealed by the (103) reciprocal space map (RSM), shown in Figure S1.D. The out-of-plane lattice parameter is calculated to be $3.863 \AA$.

To probe the volume-averaged composition of our films, we have conducted Rutherford backscattering spectrometry (RBS) measurements at PNNL. We have measured several 20$40 \mathrm{~nm}$-thick films at a $\mathrm{He}^{+}$ion energy of $4 \mathrm{MeV}$ with an incident angle of $7^{\circ}$ and a scattering angle of $170^{\circ}$, to determine the average cation composition. We then performed additional resonant elastic ion scattering (RES) measurements at a $\mathrm{He}^{+}$energy of $3.035 \mathrm{MeV}$ with an incident angle of $60^{\circ}$ and a scattering angle of $170^{\circ}$, which is much more sensitive to oxygen composition. Representative results are shown in Figures S1.E-F, along with fits to the different ion species calculated using the SIMNRA software package. From our fits to the data in Figure S1.E, we calculate a nominal composition of $19.0 \pm 0.1$ at \% La, $10 \pm 2$ at \% $\mathrm{Mn}$ and $11 \pm 2$ at \% Ni. In Figure S1.F we calculate a composition of $18.7 \pm 0.1$ at \% La, $10 \pm 2$ at $\% \mathrm{Mn}, 11 \pm 2$ at $\% \mathrm{Ni}$, and $60.0 \pm 0.1$ at $\% \mathrm{O}$. In both cases we find that the values are in excellent agreement with the expected stoichiometry, with no significant deviations in cation or oxygen composition; moreover, no changes are observed following annealing in air. 


\section{B. Crystal Orientations}

As discussed in the main text, our ordered $\mathrm{La}_{2} \mathrm{MnNiO}_{6}$ film assumes the $P 2_{1} / n$ phase at room-temperature. We note that previous studies have shown evidence for an additional $R \overline{3} c$ phase at room-temperature, ${ }^{1}$ but we do not observe any significant presence of this phase in XRD or TEM. In our work we investigate LMNO along both the pseudocubic [100] and [110] zone-axes, as shown in Figure S2; the former orientation allows us to visualize octahedral La cation displacements associated with ordering, while the latter allows us to separate $\mathrm{Mn}$ and Ni cations onto different sublattices to facilitate mapping of cation order.
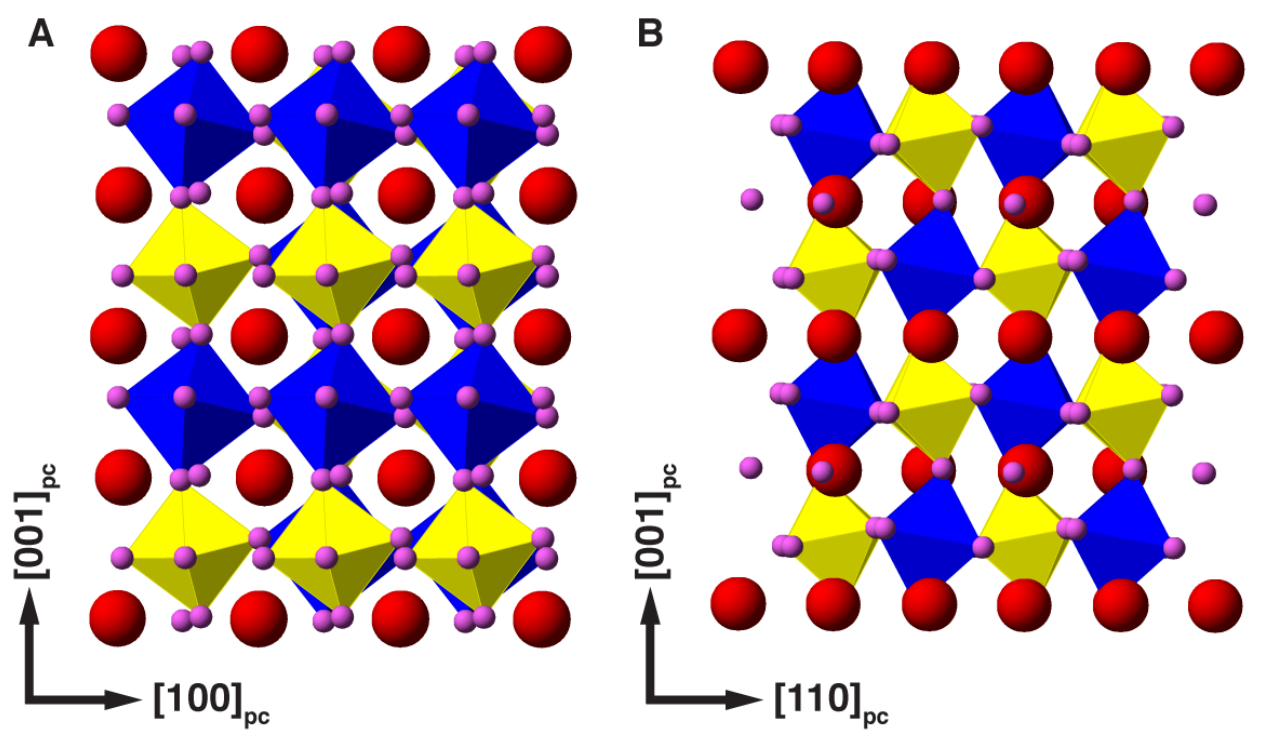

FIG. S2. LMNO crystal orientations used in this study. (A) Pseudocubic [100] zone-axis used for measuring the displacement of La cations. (B) Pseudocubic [110] zone-axis used for STEM-EDS mapping of cation ordering. 


\section{RevSTEM Interaxial Angle Measurements}

STEM offers an extremely high-resolution probe of structure and chemistry, but it is prone to scan distortions and sample drift artifacts that can compromise measurements. ${ }^{2}$ We employ a method of correcting these distortions that allows us to precisely measure pseudocubic interaxial angles at both atomic-level and intermediate magnifications; this approach, called Revolving STEM (RevSTEM), ${ }^{3}$ utilizes a time-series of scan-rotated image frames to correct scanning distortions, enabling measurements of atomic displacements with picometer precision.

The RevSTEM measurements described in the main text reveal a regular distribution of La - La - La interaxial angles, in excellent agreement with the theoretical LMNO crystal structure. Figure S3 shows the histograms of interaxial angles for purely ordered regions at high magnification, as well for a mix of regions at lower magnification. In the former case there is a pattern of angles distributed about $92.6 \pm 0.1^{\circ}$ and $87.5 \pm 0.1^{\circ}$, very close to the expected values of $92.67^{\circ}$ and $87.99^{\circ}$ calculated from the bulk crystal structure. In contrast, the distribution at lower magnification includes additional angles in between these extremes, resulting from structural disorder.

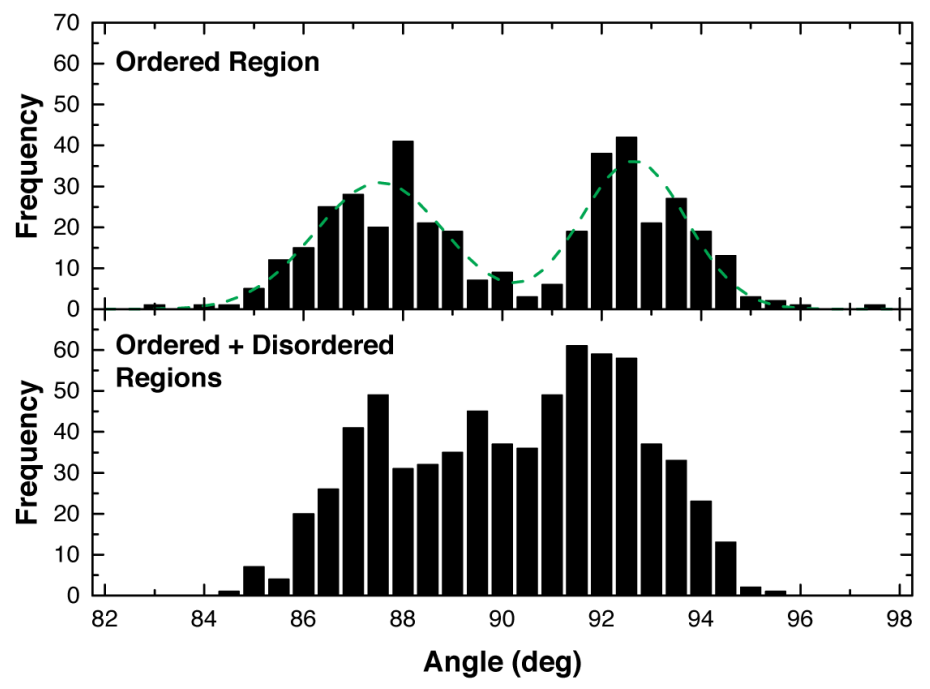

FIG. S3. Distribution of La - La - La interaxial angles measured by RevSTEM. Top: Distribution of angles measured for a purely ordered region at high-magnification, overlaid with Gaussian fits to the data. Bottom: Distribution of angles measured at intermediate-magnification for a mix of ordered and disordered regions. 


\section{STEM-EDS Measurements of NiO Secondary Phase}

We have conducted further STEM-EDS analysis to quantify the composition of the $\mathrm{NiO}$ secondary phase and matrix. We use the COMPASS MSA package (part of the ThermoFisher Noran 7 program), which improves the signal-to-noise of our maps and allows us to automatically identify meaningful physical phases. ${ }^{4}$ In this way we are able to spatially separate any peak overlaps without any user intervention. Our results, shown in Figure S4 and Table SI for the as-grown film, indicate a sizable volume fraction of $\mathrm{NiO}$ regions, in agreement with the discussion in the main text. From the COMPASS analysis we estimate the composition of the matrix as $20.9 \pm 0.2 \mathrm{La}, 9.4 \pm 0.1 \mathrm{Mn}, 6.6 \pm 0.1 \mathrm{Ni}$, and $60.6 \mathrm{O}$ at $\%$, while the composition of the secondary phase is $17.7 \pm 0.2 \mathrm{La}, 8.7 \pm 0.1 \mathrm{Mn}, 11.1 \pm 0.1$ $\mathrm{Ni}$, and $59.6 \mathrm{O}$ at \%. After renormalizing the $\mathrm{La}, \mathrm{Mn}$, and $\mathrm{Ni}$ compositions independently of $\mathrm{O}$, as in the main text, we calculate cation ratios of $\mathrm{La} 0.57$, Mn 0.26 , and $\mathrm{Ni} 0.18$ for the matrix and La 0.47 , Mn 0.23, and Ni 0.30 for the secondary phase. This result reveals that the average composition of the matrix is Ni-deficient, while the secondary phases exhibit a near-doubling of $\mathrm{Ni}$ content, in agreement with the formation of a $\mathrm{NiO}$ phase. We note that these numbers must be viewed with caution because of the overlap between the secondary phase and matrix. Nonetheless, we find that our STEM-EDS and APT results are in good agreement, supporting both the formation of $\mathrm{NiO}$ secondary phases and a Mn-rich matrix. 

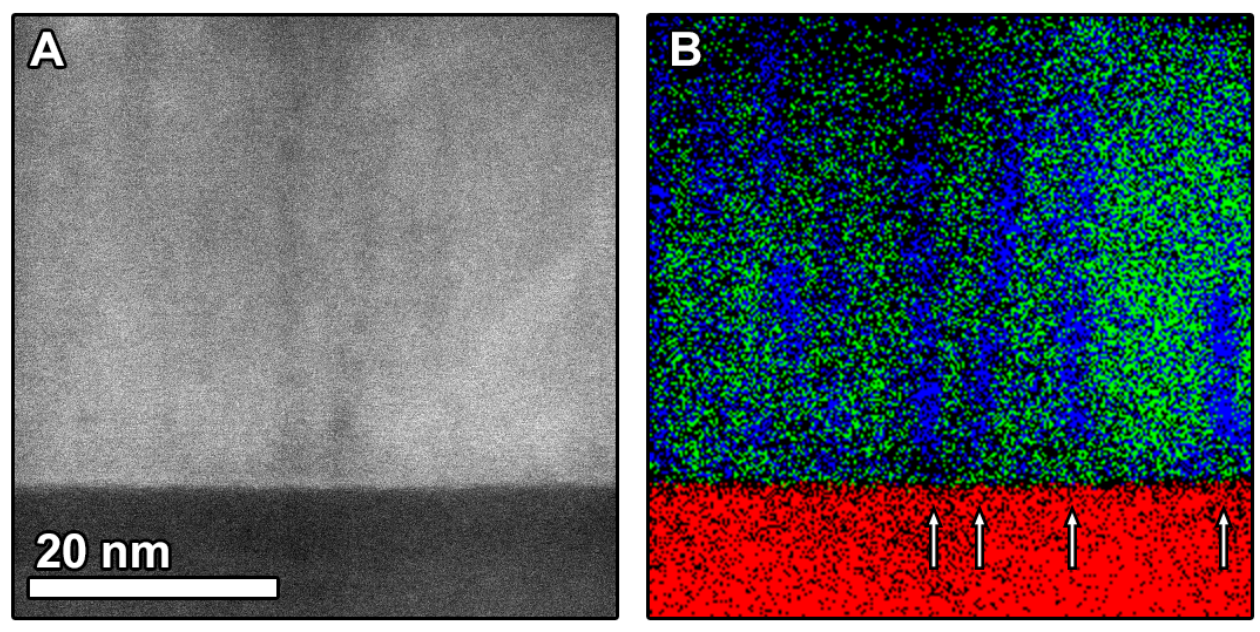

FIG. S4. STEM-EDS map of the annealed film processed using COMPASS. (A) Representative STEM-HAADF image of the annealed film used for EDS mapping. (B) Phase map with red, green, and blue corresponding to the STO substrate, LMNO matrix, and NiO precipitates (marked with arrows), respectively.

\begin{tabular}{|c|c|c|c|c|c|}
\hline \multirow{2}{*}{ Sample } & \multirow{2}{*}{ Phase } & \multicolumn{4}{|c|}{ Composition (At \%) } \\
\hline & & $\mathrm{La}$ & Mn & $\mathrm{Ni}$ & $\mathrm{O}$ \\
\hline \multirow{2}{*}{ As-Grown } & Matrix & $20.9 \pm 0.2$ & $9.4 \pm 0.1$ & $6.6 \pm 0.1$ & 60.6 \\
\hline & Secondary Phase & $17.7 \pm 0.2$ & $8.7 \pm 0.1$ & $11.1 \pm 0.1$ & 59.6 \\
\hline \multicolumn{2}{|c|}{ Nominal Film Composition } & 20 & 10 & 10 & 60 \\
\hline
\end{tabular}

TABLE SI. Matrix and NiO secondary phase compositions measured by STEM-EDS. The secondary phase is significantly enriched in $\mathrm{Ni}$, while the surrounding matrix is enriched in Mn. 


\section{E. Crystallography of the NiO Secondary Phase}

We have conducted additional STEM-HAADF imaging to explore the orientation relationship between the $\mathrm{NiO}$ phase and the LMNO matrix. Figure S5 shows the case where a $\mathrm{NiO}$ particle has penetrated the surface of the LMNO film. Fast Fourier transforms (FFTs)

of both phases shows that they appear to possess an epitaxial $\mathrm{NiO}$ [110] // LMNO [110] orientation relationship. We find that both phases are close to their bulk pseudocubic lattice parameters of $4.17 \AA$ and $3.87 \AA$ for $\mathrm{NiO}$ and LMNO, respectively; ${ }^{5,6}$ in this case we expect a rather large out-of-plane misfit strain between the phases of $\sim 7.2 \%$. We observe significant lattice plane bending around the particle, as well as FFT frequency splitting, which suggest the strain is at least partly accommodated by misfit dislocations.

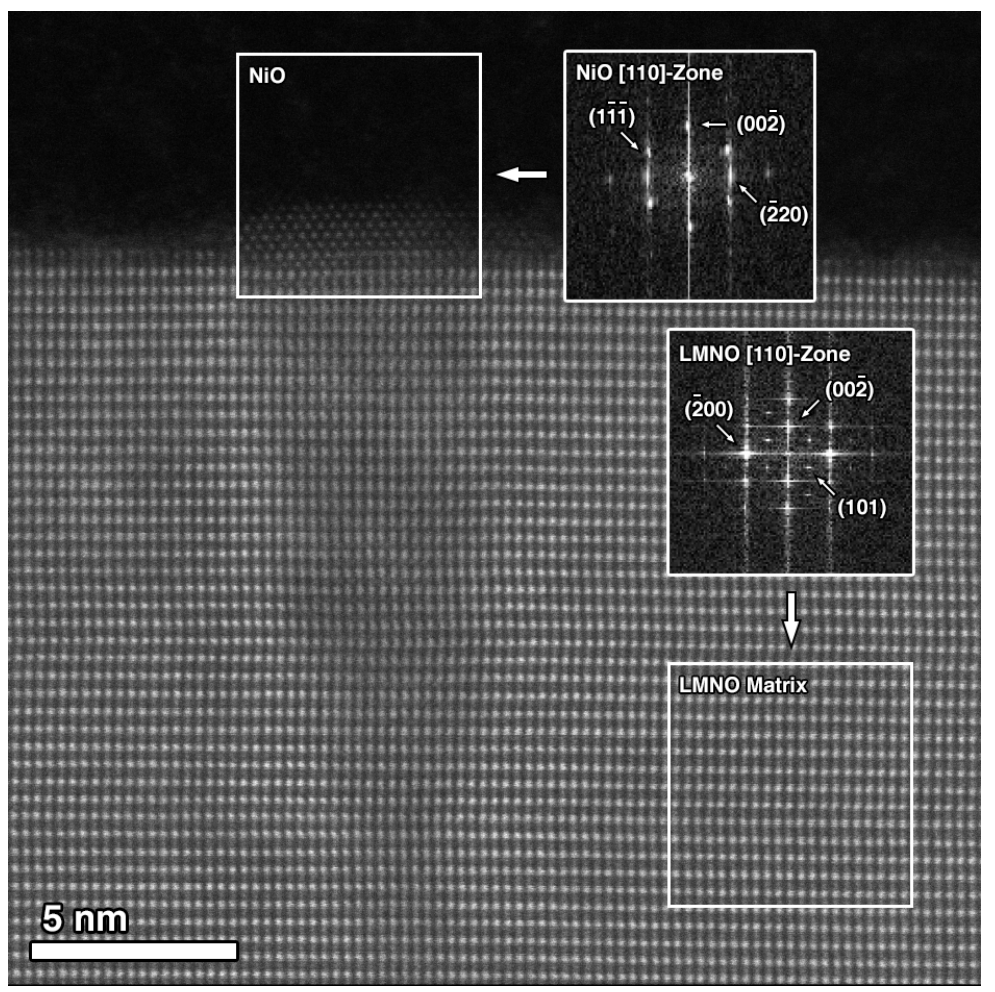

FIG. S5. NiO secondary phase orientation. Representative STEM-HAADF image taken along the LMNO [110] zone-axis showing a NiO particle emerging from the surface of the film. The inset FFTs show that both the particle and matrix are [110]-oriented. This orientation relationship is also present in other parts of the film. 


\section{F. STEM-EELS Analysis of NiO Secondary Phase Environment}

To explore chemistry changes in the vicinity of the $\mathrm{NiO}$ secondary phase, we have conducted electron energy loss spectroscopy (EELS) measurements. We are particularly interested in local Mn valence changes, since these may be associated with a spin state transition and a reduction in FM ordering. ${ }^{7}$ Figure $\mathrm{S} 6$ shows EELS maps in the vicinity of a $\mathrm{NiO}$ particle, taken from the representative region marked in Figure S6.A. Because of the overlap of the La $M_{45}$ and $\mathrm{Ni} L_{23}$ edges we have conducted multiple linear least squares (MLLS) fitting using reference spectra taken from a bulk $\mathrm{LaCrO}_{3}$ standard sample prepared via MBE and a bulk NiO standard in the Digital Micrograph EELS Atlas. After fitting the LMNO matrix and $\mathrm{NiO}$ secondary phase are clearly separated, as shown in Figures S6.C-F. We note the presence of a residual $\mathrm{Mn}$ signal in the $\mathrm{NiO}$ core, owing to the projection of the small precipitate $(\sim 2 \mathrm{~nm}$ diameter) onto the surrounding matrix. Nonetheless, this partitioning agrees well with both our STEM-EDS and APT results.

Having confirmed the configuration of the $\mathrm{NiO}$ secondary phase environment, we now
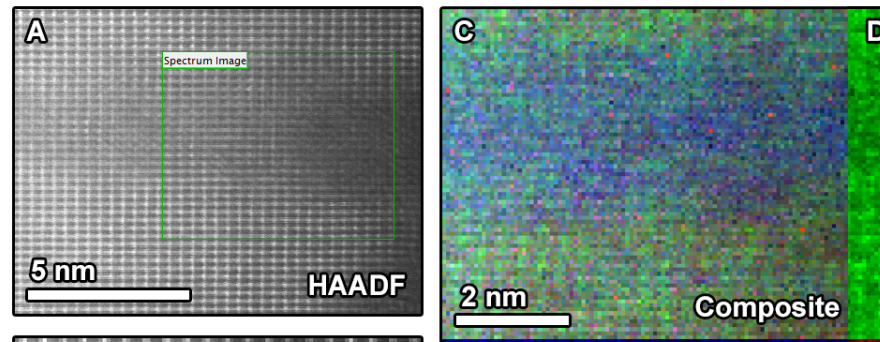

D

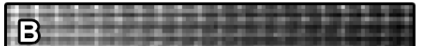

E
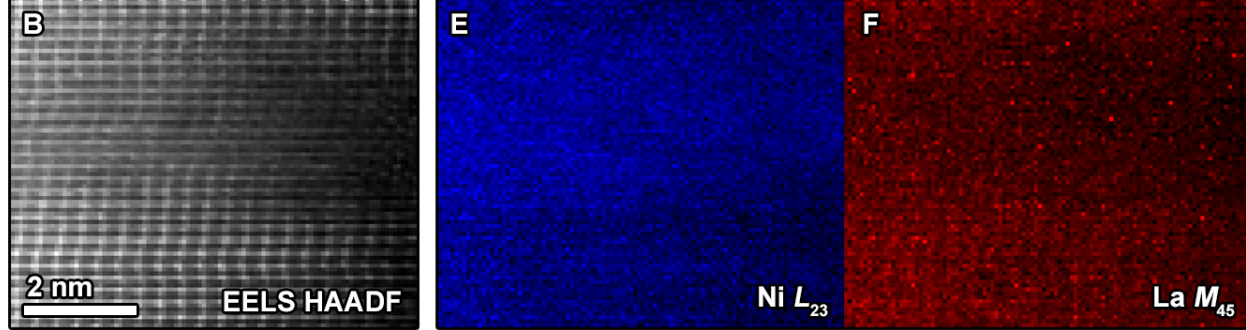

FIG. S6. STEM-EELS mapping of composition in the vicinity of a NiO region. (A) STEM-HAADF survey image of the annealed film used for EELS mapping. (B) STEM-HAADF image acquired during EELS mapping. (C-F) Composition maps determined using MLLS fitting. C shows a composite of the various edges, while D-F correspond to the $\mathrm{Mn} L_{23}$, Ni $L_{23}$, and La $M_{45}$ edges, respectively. 

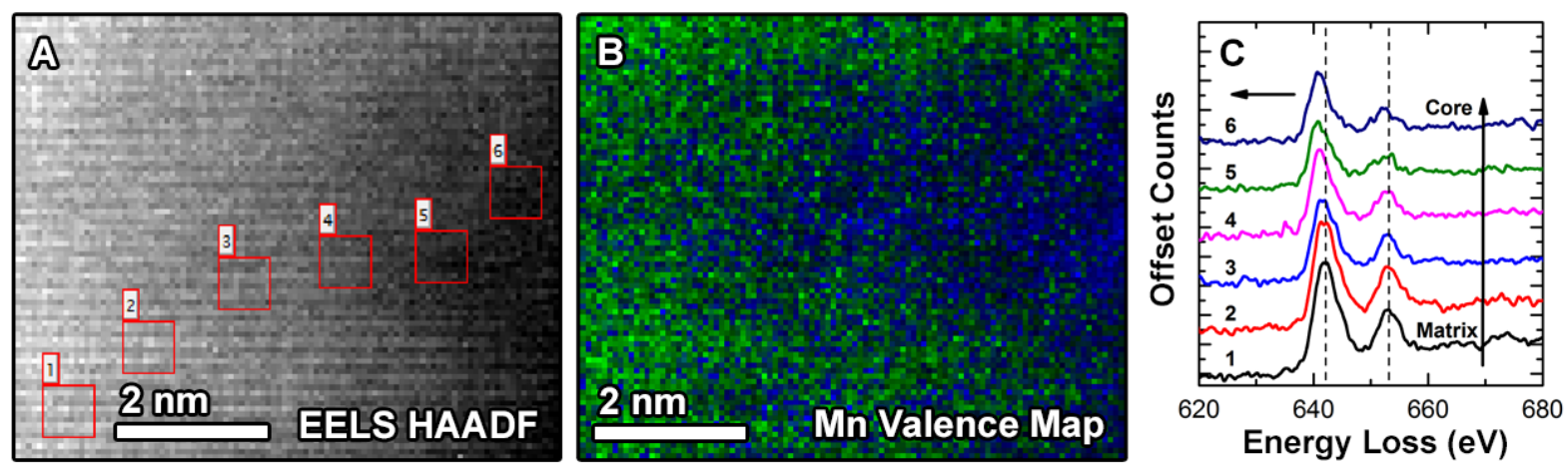

FIG. S7. STEM-EELS mapping of Mn valence in the vicinity of a NiO region. (A) EEL spectrum image overlaid with regions from which the average spectra are extracted. (B) MLLS fitting of two distinct Mn valence components. Green corresponds to a spectrum extracted from the matrix (region 1) and blue to a spectrum extracted from the core (region 6). (C) Plots of the progression of the $\mathrm{Mn} L_{23}$ edge fine structure moving from the matrix (region 1) into the NiO core (region 6). After aligning the spectra to the $\mathrm{O} K$ edge to account for any energy drift, the spectra exhibit a significant chemical shift toward lower energy loss, indicating a reduction in Mn valence. The dashed lines have been added as a guide to the eye.

turn to the $\mathrm{Mn} L_{23}$ edge. The fine structure of this edge is known to be highly sensitive to Mn valence in transition metal oxides; in particular, a chemical shift of the edge to lower energy loss is associated with a reduction in Mn valence. ${ }^{8}$ Figure S7.A shows the recorded spectrum image, overlaid with regions from which averaged spectra were extracted. To account for energy drift we have aligned the spectrum image to the known position of the $\mathrm{O}$ $K$ edge at $532 \mathrm{eV}$. We then repeated the MLLS fitting procedure, this time using reference spectra taken from regions 1 and 6 . The results of this fitting are shown in Figure S7. The map in Figure S7.B indicates a stark transition from the Mn valence environment of the matrix (green) to a different environment around the $\mathrm{NiO}$ core (blue). Individual averaged spectra, plotted in Figure S7.C, show this transition even more clearly. There is a significant chemical shift of both the $\mathrm{Mn} L_{3}$ edge $(\Delta=-1.5 \pm 0.1 \mathrm{eV})$ and $L_{2}$ edge $(\Delta=-0.9 \pm 0.1$ $\mathrm{eV}$ ) toward lower energy loss. This shift is in excellent agreement with literature values, ${ }^{8}$ indicating a change from the nominal $\mathrm{Mn}^{4+}$ valence in the matrix toward a more $\mathrm{Mn}^{3+}-$ like state around the $\mathrm{NiO}$ core. Such a Mn valence will greatly affect the magnetic ordering of the system, reducing the effective spin state of the ion and inhibiting superexchange. 


\section{G. DFT Calculations}

The thermodynamic stability of the ordered double-perovskite LMNO and its disproportionated phases was evaluated with respect to the binary oxides $\mathrm{La}_{2} \mathrm{O}_{3}, \mathrm{MnO}_{2}$ (rutile phase) and $\mathrm{NiO}$ as:

$$
\begin{array}{r}
\Delta G=E\left(\mathrm{LMNO}^{\prime}\right)-\left[A \times E\left(\mathrm{La}_{2} \mathrm{O}_{3}\right)+\right. \\
B \times E\left(\mathrm{MnO}_{2}\right)+C \times E(\mathrm{NiO}) \\
\left.+(D / 2) \times E\left(\mathrm{O}_{2}\right)-D \times \Delta \mu_{\mathrm{O}}\right]
\end{array}
$$

where LMNO' indicates a generally disordered and non-stoichiometric phase of $\mathrm{La}_{2} \mathrm{MnNiO}_{6}$ or a combination of several such phases and $\mathrm{NiO}$. Coefficients $A, B$, and $C$ define the relative weight of the corresponding binary oxides. They are determined as the ratio of La:Mn:Ni is $2: 1: 1$, in accordance with the volume-averaged experimental data. The value of $D$ defines the excess of oxygen in LMNO with respect to the binary oxides; this number is negative if the LMNO phase is oxygen-deficient with respect to the combination of the binary oxides. Finally, $\Delta \mu_{\mathrm{O}}$ is an independent parameter corresponding to the oxygen chemical potential. To relate $\Delta \mu_{\mathrm{O}}$ to the experimental conditions we approximate it as:

$$
\Delta \mu_{\mathrm{O}}=1 / 2\left[\Delta G_{\text {gas }}\left(\mathrm{O}_{2}\right)\left(T, p^{0}\right)+k T \times \ln \left(p / p^{0}\right)\right.
$$

where $\Delta G_{\text {gas }}\left(\mathrm{O}_{2}\right)\left(T, p^{0}\right)$ is the variation of Gibbs free energy for oxygen gas at standard pressure $p^{0}=1$ atm with respect to its value at $0 \mathrm{~K}$, and $T$ and $p$ are the growth temperature and $\mathrm{O}_{2}$ pressure, respectively.

Among the considered cases (see Table SII), the most thermodynamically favorable LMNO phases are:

- $\mathrm{La}_{2} \mathrm{MnNi}_{1-x} \mathrm{O}_{6-x}$

- $\mathrm{La}_{2} \mathrm{Mn}_{1+x} \mathrm{Ni}_{1-2 x} \mathrm{O}_{6} \& \mathrm{La}_{2} \mathrm{Mn}_{1-y} \mathrm{Ni}_{1+y} \mathrm{O}_{6-y}$

- $\mathrm{La}_{2} \mathrm{Mn}_{1+x} \mathrm{Ni}_{1-x} \mathrm{O}_{6}$

shown as cases 1,2, and 3, respectively in main text Figure 5. Here we consider situations where $x=y$, though we note that this is not necessarily a requirement. 


\begin{tabular}{lccc}
\hline \hline Double perovskite and other LMNO phases & $x / y$ & Description \\
\hline $\mathrm{La}_{2} \mathrm{MnNiO}_{6}$ & - & - & Ideal LMNO \\
$\mathrm{La}_{2} \mathrm{MnNiO}_{6-x}$ & - & 0.125 & $\mathrm{O}$ Vacancy $\left(V_{\mathrm{O}}\right)$ \\
$\mathrm{La}_{2} \mathrm{MnNi}_{1-x} \mathrm{O}_{6}$ & $\mathrm{NiO}$ & 0.125 & $\mathrm{Ni}$ Vacancy $\left(V_{\mathrm{Ni}}\right)$ \\
$\mathrm{La}_{2} \mathrm{MnNi}_{1-x} \mathrm{O}_{6-x}$ & $\mathrm{NiO}$ & 0.125 & $V_{\mathrm{O}-} V_{\mathrm{Ni}}$ Pair $($ Case 1) \\
$\mathrm{La}_{2} \mathrm{MnNi}_{1-x} \mathrm{O}_{6-2 x}$ & $\mathrm{NiO}$ & 0.125 & $2 V_{\mathrm{O}}+V_{\mathrm{Ni}}$ \\
$\mathrm{La}_{2} \mathrm{Mn}_{1+x} \mathrm{Ni}_{1-2 x} \mathrm{O}_{6} \& \mathrm{La}_{2} \mathrm{Mn}_{1-y} \mathrm{Ni}_{1+y} \mathrm{O}_{6-y}$ & $\mathrm{NiO}$ & $0.125,0.25$ & Case 2 \\
$\mathrm{La}_{2} \mathrm{Mn}_{1+x} \mathrm{Ni}_{1-x} \mathrm{O}_{6}$ & $\mathrm{NiO} \& \mathrm{La}_{2} \mathrm{O}_{3}$ & $0.125,0.25$ & Case 3 \\
\hline \hline
\end{tabular}

TABLE SII. $\mathrm{La}_{2} \mathrm{MnNiO}_{6}$ and other LMNO phases considered in this study. Free energies of these phases calculated with respect to $\mathrm{La}_{2} \mathrm{O}_{3}, \mathrm{MnO}_{2}$, and $\mathrm{NiO}$ are shown in main text Figure 5. The disproportionation coefficients are omitted. 


\section{REFERENCES}

${ }^{1}$ Rogado, N. S.; Li, J.; Sleight, A. W.; Subramanian, M. A. Magnetocapacitance and magnetoresistance near room temperature in a ferromagnetic semiconductor: $\mathrm{La}_{2} \mathrm{NiMnO}_{6}$. $A d v$. Mater. 2005, 17, 2225-2227.

${ }^{2}$ Jones, L.; Nellist, P. D. Identifying and correcting scan noise and drift in the scanning transmission electron microscope. Microsc. Microanal. 2013, 19, 1050-60.

${ }^{3}$ Sang, X.; LeBeau, J. M. Revolving scanning transmission electron microscopy: Correcting sample drift distortion without prior knowledge. Ultramicroscopy 2014, 138, 28-35.

${ }^{4}$ Kotula, P. G.; Klenov, D. O.; von Harrach, H. S. Challenges to Quantitative Multivariate Statistical Analysis of Atomic-Resolution X-Ray Spectral. Microsc. Microanal. 2012, 18, 691-698.

${ }^{5}$ Bull, C. L.; Gleeson, D.; Knight, K. S. Determination of B-site ordering and structural transformations in the mixed transition metal perovskites $\mathrm{La}_{2} \mathrm{CoMnO}_{6}$ and $\mathrm{La}_{2} \mathrm{NiMnO}_{6} . J$. Phys. Condens. Matter 2003, 15, 4927-4936.

${ }^{6}$ Thomassen, L. An X-Ray Investigation of the System $\mathrm{Cr}_{2} \mathrm{O}_{3}-\mathrm{NiO}^{1}$. J. Am. Chem. Soc. 1940, 62, 1134-1136.

${ }^{7}$ Singh, M. P.; Truong, K. D.; Jandl, S.; Fournier, P. Long-range Ni/Mn structural order in epitaxial double perovskite $\mathrm{La}_{2} \mathrm{NiMnO}_{6}$ thin films. Phys. Rev. B 2009, 79, 1-6.

${ }^{8}$ Kurata, H.; Colliex, C. Electron-energy-loss core-edge structures in manganese oxides. Phys. Rev. B 1993, 48, 2102-2108. 